\title{
AVALIAÇÃO DA ROTULAGEM DE SUPLEMENTOS ENERGÉTICOS EM BRASÍLIA
}

\author{
EVALUATION OFENERGY SUPPLEMENTS LABELS IN BRASÍLIA
}

EVALUACIÓN DELOS RÓTULOS DE SUPLEMENTOS ENERGÉTICOS EN BRASÍLIA

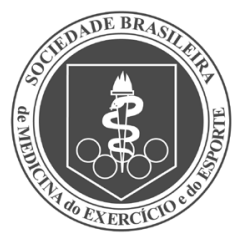

Artigo Original

Original ARticle Artículo Original

\author{
Carolina Amâncio Louly Sasaki ${ }^{1,2}$ \\ (Nutricionista) \\ Bruna Lira Mareth² \\ (Nutricionista) \\ Sandra Fernandes Arruda ${ }^{2}$ \\ (Nutricionista) \\ Teresa Helena Macedo da Costa² \\ (Nutricionista e Profissional de \\ Educação Física)
}

1. Centro Universitário Unieuro, Departamento de Nutrição, Brasília, DF, Brasil.

2. Universidade de Brasília, Departamento de Nutrição, Brasília, DF, Brasil.

\section{Correspondência:}

Departamento de Nutrição, Faculdade de Ciências da Saúde, Campus Universitário Darci Ribeiro, Universidade de Brasília, Brasília, DF, Brasil. 79910-900.

carolinanutricionista09@gmail.com

\section{RESUMO}

Introdução: A legislação brasileira dispõe de regulamentação para a comercialização de suplementos para atletas. Nesse contexto, um dos suplementos muito utilizados para atletas são os energéticos ricos em carboidratos, os quais são excelentes fontes de energia para o exercício. Objetivo: Avaliar a adequação da rotulagem de suplementos energéticos à base de carboidratos comercializados em Brasília, Distrito Federal, de acordo com a legislação vigente. Métodos: Foram avaliados rótulos de produtos comercializados em farmácias e lojas de suplementos nutricionais na região do Plano Piloto de Brasília. As informações avaliadas nos rótulos foram uso pretendido do produto, composição específica, presença de frase obrigatória e ausência de imagens e expressões proibidas de acordo com a Resolução da Diretoria Colegiada número 18/2010 da Agência Nacional de Vigilância Sanitária (ANVISA). Resultados: Foram catalogados 60 produtos nacionais e importados. Verificou-se que $75 \%$ da amostra apresentaram algum item em desacordo com a resolução. A presença da frase obrigatória em negrito e em destaque foi o item com maior percentual de inadequação (73,3\%). Além disso, houve casos de a declaração obrigatória estar redigida com significado oposto. Todos os suplementos atenderam à legislação no que se refere ao teor mínimo de carboidratos relativo ao valor energético total do produto e à quantidade mínima de carboidratos na porção do produto. Conclusões: Considerando-se que 75\% dos suplementos à base de carboidratos apresentaram inadequação de rotulagem e que a inadequação mais frequente foi a ausência ou redação incorreta da frase obrigatória nos rótulos dos suplementos, conclui-se que esse tipo de irregularidade atende aos interesses da indústria e não necessariamente aos interesses do consumidor. A presença da declaração obrigatória no produto é um requisito facilmente fiscalizável, portanto, destaca-se a necessidade de fiscalização na comercialização dos suplementos energéticos. Nível de Evidência Ill; Análises econômicas e de decisão - Desenvolvimento de modelo econômico ou de decisão.

Descritores: Suplementos nutricionais; Carboidratos; Informação nutricional; Legislação.

\section{ABSTRACT}

Introduction: Brazilian legislation provides regulations for the marketing of supplements for athletes. In this context one of the most widely used supplements for athletes are the carbohydrate-rich ones, which are excellent sources of energy for exercise. Objective: To evaluate the adequacy of the labeling of supplements based on carbohydrates marketed in Brasilia, Federal District, Brazil according to the current legislation. Methods: The labels of products marketed in drugstores and nutritional supplement stores were evaluated in the region of the Plano Piloto de Brasilia. The information evaluated on the labels were intended use of the product, specific composition, presence of mandatory phrase and absence of prohibited images and expressions according to the Resolution of the Collegiate Board of Directors number 18/2010 of the National Agency of Sanitary Surveillance (ANVISA). Results: Sixty national and imported products were cataloged. It was observed that $75 \%$ of the sample products had some item that failed to comply with the regulation. The presence of the mandatory statement in bold and highlighted was the item with the highest percentage of inadequacy (73.3\%). In addition, there have been cases where the mandatory statement is worded with opposite meaning. All supplements have complied with the legislation regarding the minimum carbohydrate content relative to the total energy value of the product and the minimum amount of carbohydrate per serving. Conclusions: Considering that 75\% carbohydrate-based supplements were in adequate for labeling and that the most frequent mismatch was the absence or incorrect wording of the mandatory statement on the labels of supplements, it is concluded that this type of irregularity serves the interests of industry and not necessarily the interests of the consumer. The presence of the mandatory statement in the product is an easily enforceable requirement, therefore, it is emphasized a more efficient inspection of the energy supplements marketed. Level of Evidence III; Economic and decision analyses - Developing an economic or decision model.

Keywords: Dietary supplements; Carbohydrates; Nutritional facts; Legislation.

\section{RESUMEN}

Introducción: La legislación brasileña dispone la reglamentación para la comercialización de suplementos para atletas. En este contexto, uno de los suplementos más utilizados para atletas son los energéticos ricos en carbohidratos, los cuales son excelentes fuentes de energía para el ejercicio. Objetivo: Evaluar la adecuación del rotulado de suplementos energéticos a base de carbohidratos comercializados en Brasilia, Distrito Federal, de acuerdo con la legislación vigente. 
Métodos: Fueron evaluados los rótulos de productos comercializados en farmacias e tiendas de suplementos nutricionales en la región del Plano Piloto de Brasília. Las informaciones evaluadas en los rótulos fueron uso pretendido del producto, composición específica, presencia de información obligatoria e la ausencia de imágenes y expresiones prohibidas de acuerdo con la Resolución del Directorio Colegiado número 18/2010 de la Agencia Nacional de Vigilancia Sanitaria (ANVISA). Resultados: Fueron catalogados 60 productos nacionales e importados. Se verificó que el 75\% de la muestra presentó algún ítem en desacuerdo con la resolución. La presencia de la información obligatoria en negrita een destaque fue el ítem con mayor porcentaje de inadecuación (73,3\%). Además, hubo casos de que la información obligatoria esté redactada con un significado opuesto. Todos los suplementos atendieron a la legislación en lo que se refiere al grado mínimo de carbohidratos relativo al valor energético total del producto y a la cantidad mínima de carbohidratos en la porción del producto. Conclusiones: Considerando que el 75\% de los suplementos a base de carbohidratos presentaron inadecuación de rotulado y que la inadecuación más frecuente fue la ausencia o redacción incorrecta de la información obligatoria en los rótulos de los suplementos, se concluye que ese tipo de irregularidad atiende a los intereses de la industria eno necesariamente a los intereses del consumidor. La presencia de la información obligatoria en el producto es un requisito fácilmente fiscalizable, por lo que se destaca la necesidad de fiscalización en la comercialización de los suplementos energéticos. Nivel de Evidencia Ill; Análisis económico y de decisión-Desarrollo de modelo económico o de decisión.

Descriptores: Suplementos dietéticos; Carbohidratos; Información nutricional; Legislación.

\section{INTRODUÇÃO}

Os carboidratos são considerados um dos melhores recursos ergogênicos disponíveis para atletas e praticantes de atividade física. O consumo deste macronutriente é capaz de repor os estoques de glicogênio hepático e muscular dos indivíduos ativos, resultando em melhora da capacidade de performance do exercício e das adaptações do treinamento. No caso de atletas e desportistas envolvidos em treinamento intenso, a necessidade de carboidratos pode ser consideravelmente elevada. O fornecimento adequado de carboidratos apenas pela alimentação torna-se muitas vezes inviável, pois o elevado volume e número das refeições podem interferir negativamente nos treinos. Em situações como essa, a suplementação é uma conduta recomendável. ${ }^{1,2}$ Entretanto os suplementos dietéticos nem sempre são consumidos com base nas necessidades nutricionais. De acordo com a Sociedade Brasileira de Medicina do Esporte, o Brasil tem apresentado um consumo abusivo de suplementos alimentares, especialmente em ambientes de prática de exercícios físicos e sem a devida orientação de um profissional de saúde especializado. ${ }^{3}$ Alguns estudos realizados em diferentes regiões no Brasil investigaram o consumo de suplementos alimentares em academias de ginásticas e constataram elevada prevalência de indivíduos que fazem ou já fizeram uso de algum tipo de suplemento. Na maioria das vezes, estes suplementos são indicados pelo educador físico ou autoprescrição.-6

Diante da falta de acompanhamento de um profissional habilitado para prescrever o consumo de suplementos nutricionais, os rótulos desempenham papel importante na escolha desses produtos pelos consumidores. Este fato ressalta a importância de regulamentações específicas sobre rotulagem nutricional de suplementos, a fim de reduzir os riscos à saúde relacionados ao uso desses produtos. Com base nisso, a Agência Nacional de Vigilância Sanitária (ANVISA), órgão federal responsável pela regulamentação e fiscalização de suplementos no Brasil, aprovou o Regulamento Técnico sobre Alimentos para Atletas, o qual estabelece uma série de disposições relacionadas a esses produtos. A Resolução da Diretoria Colegiada - RDC n०18/2010 da ANVISA, classifica os suplementos em seis categorias, sendo uma delas denominada "Suplemento energético para atletas", que abrange os produtos destinados a complementar as necessidades energéticas deste grupo.?

Estudos anteriores realizados no Distrito Federal avaliaram a conformidade dos rótulos de suplementos com as exigências da
Portaria no 222/1998 da ANVISA e evidenciaram que a presença de inadequações é comum. 8,9 Não encontramos trabalhos que avaliaram os rótulos de suplementos comercializados em Brasília frente a atual RDC no 18/2010. O objetivo deste trabalho foi avaliar a conformidade dos rótulos de suplementos energéticos comercializados no Plano Piloto de Brasília, Distrito Federal, de acordo com a legislação vigente.

\section{MÉTODOS}

A coleta de dados foi realizada em lojas especializadas para comercialização de suplementos nutricionais e farmácias localizadas nos bairros Asa Norte e Asa Sul de Brasília, Distrito Federal, durante o mês de maio de 2015.

A seleção dos estabelecimentos para a pesquisa foi feita com base em um levantamento online de lojas especializadas e farmácias, através dos sites "Guia Mais", "Telelista" " "Qonde", que resultou em 36 lojas especializadas - sendo 17 na região da Asa Norte e 19 na Asa Sul, e 121 farmácias - 52 farmácias na Asa Norte e 69 na Asa Sul. A seleção foi realizada incluindo-se as lojas com maior estoque e capacidade de distribuição, evitando estabelecimentos próximos, excluindo-se as filiais da mesma rede de farmácias ou loja de suplemento. Foram incluídas sete lojas especializadas e três farmácias de cada região, resultando em 20 pontos de venda.

Durante as visitas aos estabelecimentos selecionados, todos os suplementos à base de carboidratos foram catalogados e fotografados para posterior análise da adequação do rótulo com a legislação RDC no 18/2010. No caso de produtos similares, com mesma composição e fabricante, diferindo apenas em sabor, optou-se por considerar apenas um produto, independente do sabor.

Como a resolução no 18/2010 categoriza e utiliza o termo "Suplemento energético para atletas" para os produtos à base de carboidratos, buscaram-se todos os produtos com essa denominação no rótulo, assim como produtos sem essa declaração, mas que eram comercializados com o intuito de fornecer carboidratos à dieta do consumidor. Entretanto, produtos que apresentavam designação de outra categoria apresentada nesta resolução, como "Suplemento hidroeletrolítico para atletas" e "Suplemento para substituição parcial de refeições de atletas", foram excluídos da pesquisa.

O primeiro item analisado foi a presença da designação "Suplemento energético para atletas" no rótulo desses produtos, conforme disposto no Art. 50, parágrafo único, da resolução. A segunda avaliação 
comparou os rótulos coletados com os requisitos específicos para os suplementos energéticos para atletas dispostos no Art. 70 da Resolução, especificamente os incisos I, II e V, que determinam:

Pelo menos 75\% do valor energético total do produto pronto para o consumo devem ser provenientes de carboidratos;

A porção pronta para o consumo do produto deve conter, no mínimo, $15 \mathrm{~g}$ de carboidratos;

O produto não pode ser adicionado de fibras alimentares e outros não nutrientes.

Para esta análise, foi levada em consideração a tolerância de 20\% para mais ou para menos, disposta no Regulamento Técnico sobre Rotulagem Nutricional de Alimentos Embalados - RDC n 360/2003.12

A terceira análise baseou-se no Capítulo V da RDC 18/2010, que dispõe sobre a rotulagem. Foi verificada a conformidade dos rótulos referente aos artigos 21 e 27, que dispõem, respectivamente, sobre a presença da frase obrigatória em destaque e negrito: "Este produto não substitui uma alimentação equilibrada e seu consumo deve ser orientado por nutricionista ou médico" e a ausência de imagens e expressões que possam induzir o consumidor ao engano, ou imagens ou expressões com referência a hormônios e substâncias farmacológicas, ou ainda expressões como "anabolizante", "hipertrofia muscular", "queima de gordura", "anticatabólico" e outras sinônimas.

Os suplementos foram classificados em três categorias:

1. Atende a RDC integralmente, quando todos os itens estivessem em conformidade;

2. Atende a RDC parcialmente, quando apenas um item não estivesse conforme;

3. Não atende a RDC, quando dois ou mais itens apresentaram-se inadequados.

Nesta pesquisa não houve necessidade de parecer do comitê de ética e pesquisa, pois o instrumento de trabalho foi a análise de rotulagem de suplementos alimentares.

\section{RESULTADOS}

Foram catalogados 60 suplementos à base de carboidratos, fabricados por 26 laboratórios diferentes, sendo 16 nacionais e 10 internacionais. A Tabela 1 apresenta a distribuição dos suplementos em função da origem e da conformidade em atender, atender parcialmente ou não atender a RDC n०18/2010, do total de produtos avaliados, 25\% dos rótulos atenderam a todos os itens da RDC e 75\% atenderam parcialmente ou não atenderam a RDC. Vale ressaltar que nenhum suplemento importado atendeu à RDC integralmente.

Do total de produtos catalogados, 50 suplementos apresentaram-se na forma de pó e 10 em gel. Observou-se que sete produtos em gel e nove produtos em pó não atenderam à RDC, devido a irregularidades na designação dos produtos e na ausência da frase obrigatória. (Tabela 1)

No que se refere à análise de designação do produto conforme disposto no Art. $5^{\circ}$ da RDC, 48 produtos atenderam à resolução, apresentando a frase "suplemento energético para atletas" no rótulo, seis

Tabela 1. Distribuição dos suplementos energéticos de acordo com a avaliação em atender a RDC 18/2010 da ANVISA.

\begin{tabular}{c|c|c|c|c|c|c}
\hline & \multicolumn{2}{|c|}{$\begin{array}{c}\text { Origem do } \\
\text { suplemento }\end{array}$} & \multicolumn{2}{c|}{$\begin{array}{c}\text { Tipo de } \\
\text { apresentação dos } \\
\text { suplementos }\end{array}$} & \multicolumn{2}{c}{$\begin{array}{c}\text { Avaliação } \\
\text { do rótulo do } \\
\text { suplemento }\end{array}$} \\
\hline Avaliação & Nacional & Importado & Pó & Gel & Total & $\%$ \\
\hline Atende a RDC integral & 15 & 0 & 13 & 2 & 15 & 25 \\
\hline Atende a RDC parcial* & 21 & 8 & 28 & 1 & 29 & 48 \\
\hline Não Atende a RDC & 11 & 5 & 9 & 7 & 16 & 27 \\
\hline Total & 47 & 13 & 50 & 10 & 60 & 100 \\
\hline
\end{tabular}

* Atende a resolução exceto em um dos itens avaliados. Siglas: RDC - Resoluçăo da Diretoria Colegiada; ANVISA - Agência Nacional de Vigilância Sanitária. apresentavam outro tipo de designação, como"repositor energético para atletas" e"composto líquido pronto para o consumo", e seis suplementos não continham qualquer designação.

Ao analisar os requisitos específicos apresentados no Art. 70 da RDC, observou-se que todos os produtos atingiram o mínimo de $75 \%$ do valor energético proveniente de carboidratos, assim como o mínimo de $15 \mathrm{~g}$ de carboidratos na porção. Nove suplementos apresentaram quantidade de fibras alimentares maior que 0,3g na porção e um suplemento não declarou a quantidade. Entretanto, apenas dois produtos dos nove produtos que informavam haver fibras na composição listavam a presença de fibra de adição na lista de ingredientes. (Tabela 2)

Na avaliação dos itens do Capítulo V, observou-se que para o Art. 21, referente à presença da frase obrigatória em destaque e negrito, apenas 16 rótulos atenderam a RDC. Além disso, observou-se em dois produtos nacionais do mesmo fabricante a presença de frase com sentido oposto: "Este produto substitui uma alimentação equilibrada e seu consumo deve ser orientado por nutricionista ou médico". (Tabela 2)

No que se refere ao Art. 27 da RDC, foi possível identificar cinco produtos com imagens e ou expressões que podem induzir o consumidor a engano, sendo três importados e dois nacionais. As expressões encontradas foram:" anticatabolic","ganho de peso"," muscle mass gainer", "Hiper Mass Gainer" e "weight gainer".

Em geral, foi possível verificar que o item com maior irregularidade refere-se à presença da frase obrigatória em destaque e em negrito, pois $73,3 \%$ dos produtos não atenderam à RDC. O item com segundo maior percentual de irregularidade foi referente à designação "Suplemento Energético para Atletas", com 20\%. (Tabela 2)

\section{DISCUSSÃO}

O percentual de inadequações encontrados nesta análise foi semelhante ao obtido por Feitosa et al. ${ }^{10}$ que, ao avaliar 81 rótulos de alimentos para atletas comercializados frente à RDC n¹8/2010, identificaram que mais de $70 \%$ dos rótulos apresentavam pelo menos um item irregular. Para Moreira et al., ${ }^{11}$ que avaliaram a classificação de 28 suplementos esportivos quanto a conformidade da logomarca, a

Tabela 2. Quantificação das adequações e inadequações nos suplementos energéticos conforme os artigos da RDC da ANVISA.

\begin{tabular}{|c|c|c|c|c|c|c|c|c|c|}
\hline \multirow{3}{*}{$\begin{array}{c}\text { Artigo da RDC } \\
18 / 2010\end{array}$} & \multicolumn{4}{|c|}{ Adequação } & \multicolumn{4}{|c|}{ Inadequação } & \multirow{3}{*}{ Total } \\
\hline & \multicolumn{2}{|c|}{ Nacional } & \multicolumn{2}{|c|}{ Importado } & \multicolumn{2}{|c|}{ Nacional } & \multicolumn{2}{|c|}{ Importado } & \\
\hline & Pó & Gel & Pó & Gel & Pó & Gel & Pó & Gel & \\
\hline $\begin{array}{c}\text { Art. } 5 \text { - Designação } \\
\text { (suplemento energéti- } \\
\text { co para atleta) } \\
\end{array}$ & 35 & 3 & 10 & 0 & 4 & 5 & 1 & 2 & 60 \\
\hline $\begin{array}{c}\text { Art. } 7 \text { - Quantidade } \\
\text { mínima de carboidrato }\end{array}$ & 39 & 8 & 11 & 2 & 0 & 0 & 0 & 0 & 60 \\
\hline $\begin{array}{c}\text { Art. } 7 \text { - Não pode ser } \\
\text { adicionado de fibras ou } \\
\text { omissão } \\
\end{array}$ & 39 & 8 & 9 & 2 & 0 & 0 & 2 & 0 & 60 \\
\hline $\begin{array}{c}\text { Art. } 21 \text { - Frase obri- } \\
\text { gatória destaque e } \\
\text { negrito (Este produto } \\
\text { não substitui uma } \\
\text { alimentação equili- } \\
\text { brada e seu consumo } \\
\text { deve ser orientado } \\
\text { por nutricionista ou } \\
\text { médico) } \\
\end{array}$ & 13 & 2 & 1 & 0 & 26 & 6 & 10 & 2 & 60 \\
\hline $\begin{array}{c}\text { Art. } 27 \text { - Imagens ou } \\
\text { expressões induzindo } \\
\text { ao engano }\end{array}$ & 37 & 8 & 8 & 2 & 2 & 0 & 3 & 0 & 60 \\
\hline
\end{tabular}

Siglas: RDC, Resolução da Diretoria Colegiada. ANVISA, Agência Nacional de Vigilância Sanitária. 
presença de frases obrigatórias e a ausência de expressões proibidas nos rótulos de acordo com a legislação vigente, identificaram irregularidade em pouco mais de $32 \%$ da amostra analisada. Entretanto é importante notar que essas pesquisas analisaram a conformidade dos rótulos de suplementos de diversas categorias e os estudos que analisaram especificamente os suplementos à base de carboidratos basearam-se na antiga legislação., 8 Desta forma, a presente pesquisa é a primeira a avaliar a adequabilidade da rotulagem de suplementos energéticos frente à RDC no18/2010.

Existe uma grande variedade de suplementos esportivos à base de carboidratos sendo comercializados atualmente e a adequada classificação desses produtos colabora para auxiliar os consumidores no momento da compra. Entretanto a classificação apresentada pela legislação não foi compatível com a realidade comercial. Os suplementos à base de carboidratos eram frequentemente comercializados nas seções de hipercalóricos, pré-treinos, pós-treinos, repositores e energéticos, as quais também apresentavam produtos de outras categorias da RDC no18/2010, como "Suplemento para substituição parcial de refeições de atletas" e "Suplemento hidroeletrolítico para atletas". A separação dos produtos de acordo com a sua denominação contida na RDC no 18/2010 efetivamente auxilia o consumidor.

Além da falta de padronização para a comercialização dos suplementos esportivos, muitos produtos não possuem a designação conforme estabelecido pela RDC n¹8/2010. No estudo de Moreira et al., ${ }^{11}$ analisou-se a adequação à legislação vigente no que se refere à realização da mudança da classificação dos suplementos para atletas e constatou-se que 7,1\% dos produtos analisados não haviam alterado a classificação, apresentando a designação antiga. Na presente pesquisa, 20\% da amostra não estava sendo comercializada com a designação de "Suplemento energético para atletas". Para aqueles que apresentaram a denominação de "Repositor energético para atletas", como consta na Portaria n²22/1998 ${ }^{14}$, deve-se ressaltar que a atual legislação determinou o prazo de dezoito meses para que as empresas se adequassem às mudanças. ${ }^{7}$ Diante do término deste prazo, esses suplementos estão sendo comercializados indevidamente, assim como os que não apresentam designação alguma.

Ao analisar os requisitos específicos dos suplementos energéticos apresentados na legislação, observou-se que todos os produtos atenderam à RDC para os itens que exigiam teor mínimo de 75\% de carboidratos sobre o valor energético total e quantidade mínima de $15 \mathrm{~g}$ de carboidratos na porção. Entretanto, estudos realizados em Brasília, com base na Portaria n²22/1998, apresentaram produtos com conteúdo de carboidratos inadequado., ${ }^{8,9} \mathrm{~A}$ adequabilidade encontrada na presente pesquisa pode, portanto, estar relacionada ao fato de que a regulação vigente reduziu o teor mínimo de carboidrato para 75\% do valor energético total, ${ }^{7}$ enquanto a Portaria 222/1998 exigia o mínimo de 90\%.14

No que se refere ao item sobre o teor de fibras, foi possível observar que dois suplementos continham adição de fibras. Entretanto, deve-se notar que ambos os produtos são de origem importada e a adequação no teor de carboidratos não os enquadraria entre os "Suplementos para Substituição Parcial de Refeições", categoria que permite o acréscimo de fibras no produto, mas delimita a quantidade de carboidrato a 50-70\% do valor energético total.? Este fato demonstra que, as diferenças nas regulamentações de diferentes países dificultam a adequação de alguns suplementos com a legislação brasileira, mas que, ainda assim, é possível encontrar esses produtos no mercado. Deve-se ressaltar, porém, que a presença de inadequações quanto à composição dos suplementos energéticos foi menor quando comparado aos suplementos de outras categorias da legislação. No estudo de Ferreira, ${ }_{13}^{13}$ ao analisar 264 rótulos de suplementos proteicos ou de creatina para atletas comercializados em Brasília com base na Resolução no 18/2010 observou-se que 23\% não se adequavam a legislação e 10\% eram produtos com comercialização proibida no Brasil. Entre os suplementos de creatina 44\% não se adequavam à RDC 18/2010. No caso dos suplementos proteicos e de creatina avaliados por Ferreira a principal inadequabilidade foi quanto à quantidade percentual de proteína nos suplementos proteicos e menor quantidade de creatina por dose nos suplementos de creatina. Desta forma os suplementos proteicos comercializados em Brasília apresentam formulações que não atendem a composição exigida na legislação e o mais grave é a comercialização de produtos com composição proibida pela RDC no 18/2010.

No que se refere às exigências de rotulagem, a presença da frase obrigatória em negrito e destaque apresentou-se como o item com maior quantidade de inadequação nesta pesquisa. Nota-se ainda que o percentual de inadequação encontrado na presente pesquisa (75\%) é consideravelmente maior daquele obtido por Moreira et al., ${ }^{11}$ o qual identificou que $25 \%$ da amostra de suplementos em geral não atendiam a este item da RDC 18/2010. A ausência deste alerta nos rótulos pode induzir uma falsa ideia aos consumidores de que suplementos alimentares substituem adequadamente os alimentos e são suficientes para suprir as necessidades nutricionais. Esta situação torna-se ainda mais complexa diante da facilidade para aquisição desses produtos, que não exige prescrição médica ou de nutricionista. Além disso, a ausência da frase ou presença de frase com sentido oposto pode ser interpretada como um estímulo ao uso de suplementos. Entretanto, a Agência Nacional de Vigilância Sanitária e a Sociedade Brasileira de Medicina do Esporte advertem que a indicação para uso de suplementos deve ser restrita a atletas em situações específicas, visto que uma alimentação balanceada é capaz de suprir as necessidades energéticas e de nutrientes de grande parte dos atletas, e supre efetivamente dos praticantes de atividades física, dispensando o uso de suplementos.

A presença de expressões ou imagens proibidas como "hipertrofia muscular", "anticatabólico", "anabólico", entre outras, consiste em outro aspecto comum nos rótulos de suplementos esportivos. No estudo de Moreira et al., ${ }^{11}$ 14,2\% dos suplementos esportivos apresentaram alguma expressão que poderia induzir o consumidor ao erro. Ferreira, ${ }_{1}^{13}$ registrou a presença dessas expressões em $25 \%$ dos suplementos proteicos ou de creatina. Entretanto outros estudos realizados em Brasília, que analisaram a presença de expressões proibidas nos rótulos de repositores energéticos com base na resolução anterior (n 222/1998), identificaram menor percentual de produtos não conforme. ${ }^{8,9}$ Diante do baixo percentual de não conformidade apresentado pela presente pesquisa (8,3\%), assim como pelos demais estudos, sugere-se que as expressões proibidas comumente apresentadas em rótulos de suplementos estejam mais associadas aos suplementos proteicos, visto que existe um apelo da mídia associando proteínas e massa muscular.

As inadequações encontradas nos rótulos dos suplementos energéticos indicam, portanto, a necessidade de maior fiscalização e controle na comercialização desses produtos. Brasília é a cidade sede do poder governamental e das agencias de fiscalização, justificando-se que aqui a fiscalização poderia ser mais efetiva que em outras cidades. No entanto não foi esta realidade que encontramos. Os rótulos são instrumentos de veiculação de informações e, de acordo com Gabriels e Lambert, ${ }^{15}$ o conteúdo dos rótulos de suplementos esportivos influenciam os consumidores no momento da compra, fato constatado ao observar que 68\% dos entrevistados que já haviam comprado suplementos nutricionais levaram em consideração as informações de rotulagem no momento de decisão. Entretanto, alguns estudos indicam que os consumidores possuem dificuldade em compreender algumas informações devido à linguagem técnica, sugerindo que as informações deveriam ser mais acessíveis. ${ }^{16}$ 
Diante disso, ações educativas visando maior esclarecimento quanto à rotulagem nutricional de suplementos alimentares, assim como educação nutricional de atletas e consumidores em geral, podem contribuir para aquisição de produtos mais adequados às necessidades individuais. ${ }^{11}$

É importante ressaltar que o presente estudo analisou apenas uma amostragem dos suplementos energéticos comercializados nas principais lojas em duas regiões da cidade de Brasília e, portanto, pode haver outros produtos que não foram submetidos à análise. No entanto a identificação de suplementos comercializados sem a devida adequação de sua rotulagem na capital do país merece a devida divulgação. Além disso, sugere-se a realização de estudos que envolvam análises químicas dos suplementos, a fim de comparar a conformidade do conteúdo nutricional dos produtos com o apresentado nos rótulos. ${ }^{13}$

\section{CONCLUSÃO}

Os rótulos dos suplementos à base de carboidratos nas regiões Asa Sul e Asa Norte da cidade de Brasília não atendem a RDC 18/2010 para 75\% dos produtos avaliados. A maior irregularidade refere-se à ausência ou redação em sentido oposto da frase obrigatória em destaque e em negrito e de designação de "Suplemento Energético para Atletas", que são informações importantes para orientar o consumidor. A ausência da frase obrigatória nos rótulos dos suplementos atende aos interesses da indústria e não necessariamente aos interesses do consumidor. Uma atitude que é facilmente fiscalizável. O aumento da fiscalização e do controle sobre os suplementos pode reduzir as irregularidades de rotulagem e disponibilizar produtos que atendam integralmente a legislação vigente. Além disso, o consumo orientado por um profissional habilitado pode adequar a real necessidade e especificidade do consumo visando alcançar os efeitos esperados com o consumo do produto.

Todos os autores declararam não haver qualquer potencial conflito de interesses referente a este artigo.

CONTRIBUICÕES DOS AUTORES: Cada autor contribuiu individual e significativamente para o desenvolvimento do manuscrito. BLM (0000-0003-1376-4408)*: redação do artigo, realização das coletas e confecção de todo o projeto de pesquisa; CALS (0000-0002-2309-4869)*: revisão da redação e montagem das tabelas; SFA (00000003-0774-8276)*: revisão do artigo e tradução para inglês do resumo; THMC (0000-002-3396-5058)*: revisão do artigo e conceito intelectual do artigo. *ORCID (Open Researcher and Contributor ID).

\section{REFERÊNCIAS}

1. Kreider RB, Wilborn CD, Taylor L, Campbell B, Almada AL, Collins R, et al. ISSN exercise \& sport nutrition review: research \& recommendations. J Int Soc Sports Nutr. 2010;7(7):1-43.

2. Hernandez AJ, Nahas RM. Modificações dietéticas, reposição hídrica, suplementos alimentares e drogas: comprovação de ação ergogênica e potenciais riscos para a saúde. Rev Bras Med Esporte. 2009;15(3 Suppl):3-12.

3. Carvalho T, Rodrigues T, Meyer F, Lancha Jr. AH, De Rose EH, Nóbrega ACL, et al. Modificações dietéticas, reposição hídrica, suplementos alimentares e drogas: comprovação de ação ergogênica e potenciais riscos para a saúde. Rev Bras Med Esporte. 2003;9(2):43-56

4. Fayh APT, Silva CVD, Jesus FRDD, Costa GK. Consumo de suplementos nutricionais por frequentadores de academias da cidade de Porto Alegre. Rev Bras Ciênc Esporte. 2013;35(1):27-37.

5. Hirschbruch MD, Fisberg M, Mochizuki L. Consumo de suplementos por jovens frequentadores de academias de ginástica em São Paulo. Rev Bras Med Esporte. 2008;14(6):539-43.

6. Moreira FP, Rodrigues KL. Conhecimento nutricional e suplementação alimentar por praticantes de exercícios físicos. Rev Bras Med Esporte. 2014;20(5):370-3.

7. Agência Nacional de Vigilância Sanitária (Brasil). Resolução RDC nª 18, de 27 de abril de 2010. Dispõe sobre alimentos para atletas. Diário Oficial da República Federativa do Brasil. [acesso em 2015 mai 5]. Disponível em: portal.anvisa.gov.br/wps/wcm/connect/52bee2804745886b91ffd53fbc4c673

8. Pinheiro MC, Navarro AC. Adequação da rotulagem nutricional de repositores energéticos comercializados no Distrito Federal. Rev Bras Nut Esp. 2008;2(9):106-18.

9. Lisbôa CCB, Liberali R, Navarro F. Avaliação da adequação da rotulagem nutricional de repositores energéticos comercializados em lojas especializadas em suplementos alimentares de Brasília-DF à legislação vigente. Rev Bras Nut Esp. 2011;5(25):14-24.

10. Feitosa GM, Caetano YR, Torres IMS, Alves VF, Garcia TA. Alimentos para atletas: qualidade das informações do rótulo. Rev Biotecnol Cienc. 2013;2(1):32.

11. Moreira SSP, Cardoso FT, Souza GG, Silva EB. Avaliação da adequação da rotulagem de suplementos esportivos. Corpus et Scientia. 2013;9(2):45-55.

12. Agência Nacional de Vigilância Sanitária (Brasil). Resolução RDC nª 360, de 23 de dezembro de 2003. Aprova regulamento técnico sobre rotulagem nutricional de alimentos embalados. Diário Oficial da República Federativa do Brasil. [acesso em 2015 mai 8]. Disponível em: portal.anvisa.gov.br/wps/wcm/ connect/ec3966804ac02cf1962abfa337abae9d/Resolucao_RDC_n_360de_23_de_dezembro_de_2003. pdf?MOD=AJPERES

13. Ferreira ACD. Adequabilidade à legislação dos suplementos proteicos e de creatina para atletas. Nutrição em Pauta. 2013;121:15-9.

14. Agência Nacional de Vigilância Sanitária (Brasil). Portaria n 222, de 24 de março de 1998. Aprova o Regulamento Técnico para Fixação de identidade e Qualidade de Alimentos para Praticantes de Atividade Física. Diário Oficial da República Federativa do Brasil. [acesso em 2015 mai 10]. Disponível em: portal. anvisa.gov.br/wps/wcm/connect/75734700474597059f4fdf3fbc4c6735/portaria_222.pdf?MOD=AJPERES

15. Gabriels G, Lambert M. Nutritional supplement products: does the label information influence purchasing decisions for the physically active? Nutr J. 2013;12(1):133.

16. Marins BR, Jacob SDC, Peres F. Avaliação qualitativa do hábito de leitura e entendimento: recepção das informações de produtos alimentícios. Ciênc Tecnol Aliment. 2008;28(3):579-85. 